\title{
DIE ROLLE DES SCHLICHTERS IM SONDER ZIVILGERICHT AM 4. SONDERN SOZIALGERICHT VON MACAPÁ-AP, AMAZÔNIA, BRASILIEN
}

\section{ORIGINALER ARTIKEL}

NETO, Dalk Dias Salomão ${ }^{1}$, SOUSA, Nicole Moreira Faria ${ }^{2}$, DENDASCK, Carla Viana ${ }^{3}$, FECURY, Amanda Alves ${ }^{4}$, OLIVEIRA, Euzébio de ${ }^{5}$, DIAS, Claudio Alberto Gellis de Mattos $^{6}$

NETO, Dalk Dias Salomão. Et al. Die Rolle des Schlichters im Sonder Zivilgericht am 4. Sondern Sozialgericht von Macapá-AP, Amazônia, Brasilien. Revista Científica Multidisciplinar Núcleo do Conhecimento. Jahrgang 06, Ed. 05, Vol. 11, S. 80-92. Mai 2021. ISSN: 2448-0959, Zugriffsverbindung: https://www.nucleodoconhecimento.com.br/gesetz/sonder-zivilgericht, DOI: 10.32749/nucleodoconhecimento.com.br/gesetz/sonder-zivilgericht

\section{ZUSAMMENFASSUNG}

Aufgrund zahlreicher sozialer Probleme wurde Gerechtigkeit von der Gesellschaft mehr gefordert. Es bestand dringender Bedarf an einem neuen Paradigma, mit der Schaffung von Sonderverfahren, die auf Vermittlung basieren, als alternatives Mittel zur Konfliktlösung als Teillösung. Die Sondergerichte haben sich seit ihrer Gründung

\footnotetext{
${ }^{1}$ Rechtsanwalt, Bachelor of Law (CEAP - Center for Higher Education of Amapá), Spezialist für Arbeitsrecht und Arbeitsprozess der Bildungseinrichtung Danosio.

${ }^{2}$ Rechtsanwalt, Bachelor of Law (CEAP - Center for Higher Education of Amapá), Spezialist für Zivilprozessrecht der Institution Dalésio Educacional.

${ }^{3}$ Theologe, PhD in Klinischer Psychoanalyse. Er arbeitet seit 15 Jahren mit wissenschaftlicher Methodik (Forschungsmethode) in der wissenschaftlichen Produktionsberatung für Master- und Doktoranden. Spezialist für Markt- und Gesundheitsforschung, Doktorand in Kommunikation und Semiotik (PUC SP).

${ }^{4}$ Biomedizin, PhD in Tropenkrankheiten, Professor und Forscher des Medical Course of Macapa Campus, Federal University of Amapa (UNIFAP), Pro-Rektor of Research and Graduate Studies (PROPESPG) der Föderalen Universität von Amapá (UNIFAP).

${ }^{5}$ Biologe, PhD in Tropenkrankheiten, Professor und Forscher des Physical Education Course, Federal University of Pará (UFPA).

${ }^{6}$ Biologe, PhD in Theorie- und Verhaltensforschung, Professor und Forscher des Chemie-Studiengangs des Instituts für Grund-, Technik- und Technologiepädagogik (IFAP) und des Graduate Program in Professional and Technological Education (PROFEPT IFAP).

RC: 85946

Zugriffsverbindung: https://www.nucleodoconhecimento.com.br/gesetz/sonder-zivilgericht
} 
durch das Gesetz 9.099/95, das sich angesichts der großen Forderungen der Klagen als äußerst wichtig für die Neuordnung der Justiz erwies, stark weiterentwickelt. Ziel dieser Arbeit war es, die Rolle des Schlichters im speziellen virtuellen Zivilgericht in der 4avara des Sonderzivilgerichts von Macapá AP, Amazônia, Brasilien zu analysieren. Es wird der Schluss gezogen, dass die Sonderjustiz von der Umsetzung über das Gesetz 9.099/95 bis zum Aufbau ihrer Grundsätze wie Verfahrensschnelligkeit, Informalität und, was auf die Verwirklichung eines schnelleren und effizienteren Prozesses hindeutet, eine historische Entwicklung war. PROJUD und TUCUJURIS durch die Computerisierung der Sondergerichte scheinen bei der Modernisierung des Prozesses wichtig zu sein, sie einfacher, schneller und für alle zugänglich zu machen und damit den technologischen Trends der Welt zu folgen. Sondergerichte als Ganzes sollten versuchen, immer mehr in Schlichter und Schlichtungsklagen $\mathrm{zu}$ investieren, wie etwa staatliche und nationale Vermittlungswochen, denn so wird es eine große Möglichkeit geben, dass Fälle in der Justiz stärker fließen.

Schlagworte: Sonderzivilgericht, Schlichtung, Schlichter.

\section{EINLEITUNG}

Aufgrund zahlreicher sozialer Probleme wurde Gerechtigkeit von der Gesellschaft mehr gefordert, da sie Probleme wie die große Anzahl von Verfahrensanforderungen, Verzögerungen bei der Beurteilung von Prozessen und Bürokratie hatte (Sena e Richa, 2011; Guimarães, 2013).

Wenn man diese Situationen mit der Schwierigkeit des Zugangs von Menschen mit wirtschaftlicher Hyposuffizienz in der Justiz hinzufügt, war es dringend notwendig, ein neues Paradigma zu entwickeln, das als Teillösung die Schaffung von Sondergerichten auf der Grundlage der Vermittlung als alternatives Mittel zur Konfliktlösung (Sérpias, 2015) darstellt.

Die Sondergerichte in Brasilien, früher Small Claims Court genannt, modellierten eine amerikanische Struktur, die als Small Claims Courts bekannt ist. Gegründet 1934 in 
New York, mit dem Ziel, zivile Anteile von geringem Wert zu genießen. Das Hauptmerkmal dieses Schnittmodells ist der Pragmatismus, der der amerikanischen Kultur eigen ist, d. h. der Konflikt steigt zu einer schnelleren, unkomplizierten und einfacheren Perspektive auf. Es war nicht notwendig, die Zahl des Anwalts, der je nach Komplexität der Situation angefordert wurde (Porto, 2008).

Dieses Modell des Gerichts erschien in Brasilien, zuerst in Rio grande do Sul, im Jahr 1982, vor der Arbeit der Schlichtungs- und Schiedsräte, mit dem Ziel der Lösung, außergerichtlich, Konflikte von kleinen Ursachen, zeigt ein großes Interesse für den Bau eines Richters (Silva, 2013).

Nachdem die Justiz 1984 im nationalen Szenario an Bedeutung gewonnen hatte, hatte sie als Meilenstein das Gesetz Nr. 7,244, das die Schaffung und das Funktionieren von Gerichten für kleine Ursachen vorsieht, konsolidiert und den Erfolg der Erfahrungen legitimiert, die mit den Schlichtungs- und Schiedsräten gesammelt wurden, die als ihre Pflicht für gleiche Gerechtigkeit sorgen (Silva, 2013).

Von diesem Zeitpunkt an beginnen im ganzen Land mehrere Sondergerichtsverfahren, die im Staatsamt durch das ordentliche Recht geregelt sind und nur zivile Ursachen von geringem wirtschaftlichem Wert abdecken, die zunächst in bis zu zwanzig (20) Mindestlöhnen festgelegt sind. So ermöglichte sie auch den finanziell größten Möglichkeiten und rechtlichen Garantien, die Justiz zu suchen, um eine friedliche Lösung ihrer Konflikte zu erreichen (Silva, 2013).

Die Bundesverfassung von 1988 hat gezeigt, dass es wirklich notwendig ist, sonderjustizvoll zu werden, und ihre Umsetzung im ganzen Land ist eine verbindliche Maßnahme, da sie bisher für Staaten fakultativ war. Der CF sicherte durch seine Art. 98, Punkt I, die Ermächtigung zur Durchführung der Sonderzivilgerichte zur Aussöhnung, Verfolgung, Beurteilung und Durchführung von Zivilsachen von geringerer Komplexität sowie spezielle Strafgerichte zur Vereinbarkeit, Beurteilung und Ausführung von Straftaten mit geringerem Anstößigkeitdurchbringen durch mündliche und sumaríssimo (die keine Verzögerung oder Formalitäten hat)-Verfahren (Sérpias, 2015) zu. 
Von der Schaffung des Ergänzenden Gesetzes Nr. 123/2006 mit dem Ziel, die Wünsche von Kleinst- und Kleinunternehmern über die Verfassung und Regulierung von ME und EVP zu befriedigen, um die Wirtschaft zu erweitern und ihr Wachstum in der brasilianischen Wirtschaft zu entwickeln. So gewannen die ME und die EPP im nationalen Szenario Raum, und aus diesem allgemeinen Gesetz wurde die gesetzliche Bestimmung über die Möglichkeit geschaffen, dass diese Arten von Unternehmen auf staatlicher Ebene das Recht haben, vor dem Sonderzivilgericht zu erscheinen und im aktiven Pol der Klage zu erscheinen (Salomão, 2001). Angesichts der Möglichkeit des Zugangs zu einem Sondergericht können diese Unternehmen ihre Aktien ohne die Anwesenheit eines Anwalts (mit einer Obergrenze für den Wert der Klage von bis zu 20 Mindestlöhnen) eingeben, wodurch ihre Ausgaben gesenkt werden. Es ist wichtig, darauf hinzuweisen, dass die Fälle vor den Sondergerichten schneller bearbeitet werden als vor dem gemeinsamen Gericht, was eine schnellere Rückforderung des Kapitals ermöglicht, das bis dahin durch zahlungsunfähige Schuldner verloren ging (Tolentino, 2011).

Im Jahr 2001, kurz nach der Schaffung des Gesetzes 9.099/95 (Sondergerichtsgesetz), wurde die Erweiterung des Zivilgerichts geschaffen, um Kleinstunternehmen in Amapa zu dienen, da sie nicht vor dem Zentralen Sondergerichtshof, der der einzige in einem Staat zu dieser Zeit in Betrieb war, als Koordinator die Magistrat Sueli Pini, derzeit Präsidentin des Gerichtshofs von do Amapá (Serra, 2015).

Bald nach der Schaffung des nationalen Statuts von ME und EPP wurde das ergänzende Staatsgesetz Nr. 044/2007 geschaffen, wo es zeigt, dass die Vorteile des Gesetzes Nr. 123/06 von der Regierung von Amapá akzeptiert wurden, der Sondergerichtshof für Kleinunternehmen und Kleinunternehmen wurde schließlich im März 2012 gegründet (Serra, 2015).

Der Mikrounternehmensgerichtshof in Amapá spielt durch die entwickelte Arbeit und die Anzahl der fruchtbaren Aussöhnungen eine relevante Rolle. Die meisten der bereits eingereichten Fälle sind auf die Genehmigung von Vereinbarungen zurückzuführen, sowohl gerichtlich als auch außergerichtlich. Erzählen vor allem mit RC: 85946 Zugriffsverbindung: https://www.nucleodoconhecimento.com.br/gesetz/sonder-zivilgericht 
Hilfe seiner Auszubildenden Mitarbeiter, die in den meisten Fällen die Schlichter in den Anhörungen sind. Es ist wichtig zu beachten, dass das 4. Gericht des Zivilgerichts von Macapa dasjenige ist, das die meisten Auszubildenden höherer Rechtsstufe hat, angesichts ihrer Partnerschaften, die als TJAP, Fecomércio, FEMICRO und SEBRAE unterzeichnet wurden (Serra, 2015).

Daher haben sich die Sondergerichte seit ihrer Schaffung durch das Gesetz 9.099/95, das sich angesichts der großen Forderungen von Klagen als äußerst wichtig für die Neuordnung der Justiz erwies, stark weiterentwickelt. Neben der Valorisierung von ME und EPP, die Vorraum vor den Sondergerichten gewinnen, um in einer würdevolleren Weise für ihre Rechte zu kämpfen, als 4. Gericht des sonderlich bürgerlichen Gerichts von Macapá (ehemaliger Hof des Mikrounternehmens und kleine Firma von Macapa), Pionier in Brasilien.

\section{ZIEL}

Zur Analyse der Rolle des Schlichters in der speziellen virtuellen Zivilgericht in der 4avara des speziellen Zivilgericht von Macapá AP, Amazônia, Brasilien.

\section{METHODE}

Quantitative und qualitative Forschung, unter Verwendung von Daten des 4. Gerichts des Sonderzivilgerichts der Stadt Macapá, Amapá, Amazônia, Brasil, Brasilien, über die Schlichtung in Gerichtsverfahren.

\section{ERGEBNISSE UND DISKUSSION}

Die Sonderzivilgerichte sind Organe, die die Ordentliche Justiz bilden, und wurden mit dem Zweck der Schlichtung, Despross, Prozess und Hinrichtung, in den Ursachen ihrer Zuständigkeit, diszipliniert durch Bundesgesetz Nr. 9.099 vom 26. September 1995 geschaffen. Die Grundlagen dieses Verfahrens orientieren sich an den Kriterien Der Ordnung, Einfachheit, Informalität, Verfahrensökonomie und Schnelligkeit, die, wann immer möglich, zur Schlichtung die Wiedergutmachung des dem Opfer erlittenen 
Schadens und die Anwendung einer Freiheitsstrafe ohne Freiheitsentzug anstreben (Neto e Júnior, 2011).

Die eigentliche Absicht der sonderlichen Zivilgerichte ist die Lösung von Problemen des Zugangs zur Justiz, die im Wesentlichen mit den Kosten und der Verzögerung von Fällen zusammenhängen, und es gibt auch zahlreiche Hindernisse, denen die Bürger, die Gerechtigkeit fordern, begegnen (Guimarães, 2013).

Die Sonderzivilrichter sind zunehmend im Leben der gesamten Bevölkerung präsent, da ihre eigene Existenz gerechtfertigt ist. Es wird durch seine moderne Art zu handeln, durch die Qualität der Dienstleistung und durch die Interessen seiner Führer, die sie immer agiler und zugänglicher macht (Bacellar, 2003).

Das Sonderzivilgericht ist zuständig für die Schlichtung, das Verfahren und das Urteil in Zivilsachen von geringerer Komplexität, sind Beispiele: Fälle, deren Wert das Vierzigfache des Mindestlohns nicht übersteigt; die in Art. 275, Punkt II der Zivilprozessordnung aufgeführten Personen; die Räumungsaktion für den eigennützen Gebrauch; Besitzklagen auf Immobilien mit einem Wert, der nicht das Vierzigfache des Mindestlohns übersteigt; außergerichtliche Vorstände (Schuldscheine, Schecks, Verträge) in Höhe des bis zum Vierzigfachen des Mindestlohns; Schadenersatz (z.B. Schäden aus Verkehrsunfällen) (Barouche, 2010).

\section{GRUNDSÄTZE VON SONDERRICHTERN}

Die Schaffung besonderer Richter ist ein wichtiger Meilenstein für die Rechtswelt in all ihren Bereichen, was es notwendig macht, die Grundsätze hervorzuheben, die diese Ordnung leiten, sowohl bei der Suche nach ihrer Bedeutung, als auch im Umfang und in der Anwendbarkeit der Rechtsnorm (Porto, 2008).

Laut Sérpias (2015) "ist der Zweck der Schaffung eines speziellen Zivilgerichts esis, die kleinen Fragen des Bürgers in der täglichen Zeit schnell und wirtschaftlich zu lösen". So wurde das Gesetz auf der Grundlage von Grundsätzen geschaffen, die mit der Erleichterung des Zugangs derer, die es anstreben, zum Recht vereinbar sind. 


\section{PRINZIP DER ORALITÄT}

Durch die moderne Form von Spezialrichtern zeichnet sich das Prinzip der Mundart durch seine einfache Anwendbarkeit aus. Dieser Grundsatz gehört zu den Generälen von Zivilverfahren, mit dem Ziel, verfahrensrechtliche Handlungen durch mündliche Mitteilung zu ergehen, obwohl diese Rechtsakte auf das Schreiben reduziert werden können (Silva, 2012).

In speziellen Höflichkeiten ist dieses Prinzip viel präsenter, und nur das Wesentliche wird auf die Dauer reduziert. Der Rest kann auf Magnetband oder gleichwertig, gemäß Artikel 13, Nr. 3 des Gesetzes Nr. 9,099/95 (Brasil, 1995) eingraviert werden. Dies zeigt, dass die Oralität das Schreiben nicht ersetzt hat, was beide Hand in Hand gehen, da es wichtig ist, den Prozess und die Umwandlung ihrer Verfahrenshandlungen in eine Klausel umzusetzen.

Die Anwendung dieses Grundsatzes zielt auch darauf ab, Dass Verfahrenshandlungen in einer einzigen Stufe oder zu ungefähren Zeiten vollzogen werden. Daher bezieht sich dieser Grundsatz direkt auf die Verfahrensgeschwindigkeit, da es keine sehr große Zeitliche Lücke zwischen den Verfahrenspraktiken geben sollte (Silva, 2012). Daher strebt dieser Grundsatz die Verfahrensvereinfachung an, um den Fortschritt des Prozesses zu erleichtern, der in seiner Schnelligkeit gipfelt.

\section{PRINZIP DER INFORMALITÄT UND EINFACHHEIT}

Schon der Name dieser Grundsätze zeigt seine Absicht, den Prozess und den Zugang zur Justiz zu erleichtern, nicht das Formblatt selbst zu halten, sondern vielmehr eine stärkere Flexibilisierung von Verfahrenshandlungen, die sie validieren, wann immer sie ihren Zweck erreichen, gemäß (Art. 13 des Gesetzes 9.099/95): "Art. 13 - Die Verfahrensakte sind gültig, wenn sie die Zwecke erfüllen, für die sie erfüllt werden, und erfüllen die Kriterien nach Art. 2 dieses Gesetzes" (Brasil, 1995)

Das Prinzip der Einfachheit ist nichts anderes als eine Entfaltung des Prinzips der Informalität, was wiederum der Versuch ist, die Gerichtsverfahren informeller zu gestalten, ohne diese Verschärfung von Formalismen und Bürokratien. Bobbio zufolge RC: 85946 Zugriffsverbindung: https://www.nucleodoconhecimento.com.br/gesetz/sonder-zivilgericht 
führte der Eifer für Verfahrensbürokratie, anstatt die volle Wirksamkeit der gerichtlichen Versorgung zu gewährleisten, zu einer Verzögerung des Verfahrens (Porto, 2008).

Ein praktisches Beispiel für diese Grundsätze ist die Art und Weise, wie die Post von juristischen Personen des Privatrechts zitiert wird, wobei die einfache Zustellung der Korrespondenz an jeden Mitarbeiter, der für den Empfang verantwortlich ist, gültig ist (Art. 18, Punkt II, des Gesetzes Nr. 9.099/95), im Gegensatz zur Zivilprozessordnung, die die Lieferung an bestimmte Personen, z. B. solche, die Verwaltungs- oder Verwaltungsbefugnisse haben, bedingt; (Art. 18 Abs. 2 des Gesetzes 9.099/95) (Brasilien, 1995): "Art. 18 - Das Angebot ist: [...] II - im Falle einer juristischen Person oder einer einzelnen Firma durch Lieferung an die für den Empfang verantwortliche Person, die notwendigerweise zu identifizieren ist".

Daher sind diese beiden Prinzipien mit dem Hauptaugenmerk, die einfachste Bevölkerung in das Forum zu bringen, da sich der am meisten benachteiligte Teil der Menschen und Laien in Rechtsangelegenheiten vor Anwälten, Richtern, Formalismen und Menschen mit weit hergeholten Vokabeln scheut.

\section{PRINZIP DER PROZEDURALEN ÖKONOMIE}

Ein weiterer Grundsatz ist die Verfahrensökonomie, die wiederum nicht nur eine Richtschnur für das Sondergericht, sondern auch für die allgemeine Theorie des gesamten Verfahrens ist, da sie damit als Hauptziel hat, so viele Ergebnisse mit möglichst niedrigen wirtschaftlichen Ausgaben zu erzielen (Sérpias, 2015).

Daher zielt dieser Grundsatz darauf ab, dass die meisten Verfahrenshandlungen in kürzester Zeit und auf möglichst wenig teure Weise vollzogen werden.

\section{PRINZIP DER VERFAHRENSGESCHWINDIGKEIT}

Was den Grundsatz der Schnelligkeit betrifft, so wird die Wirksamkeit der Justiz gewährleistet, mit dem Ziel, die Wirkungen eines Urteils beispielsweise praktisch und 
schnell und nicht langsam zu entfalten, wie es in vielen Fällen, insbesondere außerhalb der Sondergerichte, der Fall ist (Sérpias, 2015).

Sérpias (2015) "Die Verzögerung der gerichtlichen Bestimmung ist zu einer Regel geworden, wenn sie eine Ausnahme sein sollte. Die Verzögerung des Endes des Prozesses wurde institutionalisiert." Die anderen Grundsätze sind also nichts anderes als eine Umstellung auf das Prinzip der Geschwindigkeit mit dem Ziel, den Prozess schneller zu machen.

Die höchste Erwartungshaltung des Gesetzes 9.099/95 ist daher zweifelsohne das Versprechen der Schnelligkeit ohne Verstoß gegen den Grundsatz der Sicherheit der gesetzlichen Abflüsse. Mit der Absicht, den Prozess agiler und schneller zu gestalten und nur auf diese Weise seinen Hauptzweck zu erreichen, eine effiziente Antwort der gerichtlichen Versorgung der Gesellschaft.

\section{COMPUTERISIERUNG VON SPEZIELLEN ZIVILEN JUDGES- PROJURD}

Die Welt hat in den letzten Jahren einen großen technologischen Fortschritt erlebt, verbunden mit dem Mangel an physischem Raum, um zahlreiche Prozesse zu schützen, insbesondere nach der Verfassung von 1988 und der Internationalisierung der Wirtschaft mit der Schaffung des realen, Faktoren, die die Anzahl der Gerichtsverfahren ausnutzten. Damit hat die brasilianische Justiz die gesellshft PROJUDI (Digital Judicial Process) gegründet (Silva, 2012).

Der Hauptzweck der Schaffung von PROJUDI zielt darauf ab, die Justiz zu beschleunigen, Kosten zu senken, die Fähigkeit zur Bearbeitung von Maßnahmen zu erhöhen, die Arbeit der Anwälte zu erleichtern und die Qualität der Dienstleistungen für die Parteien zu verbessern. So kann es diese Regale voller Papiere beenden, da in den vergangenen Jahrzehnten die Prozesse fast handgemacht wurden, was eine Verkürzung der Wartezeit für die Bewertung von Anfragen ermöglichte, immer auf dem Wunsch, Konflikte schneller zu lösen, ohne den Parteien Schaden zuzufügen. 
Die wirkliche Revolution begann mit dem Gesetz 11.419/2006, mit der Absicht, den elektronischen Prozess zu disziplinieren, mit tiefgreifenden Änderungen in der Zivilprozessordnung und mit dem Ziel, den durch Bürokratie und die in den Prozessen immer üblichen Weg zu straffen, zu stärken, zu verkürzen. Wir sehen, dass sich zwei Bereiche mit Derinasie, Agilität und Verfahrensgeschwindigkeit deutlich weiterentwickelt haben, da die Prozesse alle über das Internet übertragen werden, ohne das Büro verlassen zu müssen (Carvalho, 2006).

In der Frage der Computerisierung ging der Zustand von Amapa weiter, im Jahr 2005, erstellt das TUCUJURIS-System, ein technologisches Gerät, das versucht, die Aktivitäten der Justiz elektronisch zu verwalten und zu entwickeln. Computerisierung von Gerichtsverfahren und Durchführung in den Justizsekretariaten sowie Speicherung der Inhalte der verschiedenen Verfahrensakte, um so eine größere Agilität der Dienstleistungen zu erreichen und das damals bestehende System zu ersetzen (Tjap, 2016).

Das TUCUJURIS-System hat sich im Laufe der Jahre verbessert, und seine Verfahren haben sich mehr und mehr verbessert, mit Ergänzungen neuer Funktionen, die es zu einer stärker integrierten Software im Rahmen der Justiz machen, seine Operationalisierung diversifizieren, mit dem Ziel, die Ausführung von Gerichtsakten durch die Server, Richter, Anwälte Verteidiger und Staatsanwälte zu verbessern, sowie eine schnellere Reaktion auf andere Mitglieder der Gesellschaft, die diesen Dienst nutzen (Tjap , 2016).

Daher ist die Computerisierung für die Aufrechterhaltung einer schnellen und effektiven Justiz unerlässlich, weil sie die Justiz modern macht und den technologischen Trends von heute folgt.

\section{SCHLICHTUNG IN DEN SPEZIELLEN JUDGES CIVEIS}

Das Schlichtungsinstitut ist der überwiegenden Mehrheit der Brasilianer bekannt. Seine Anwendung im Bereich des Rechts, die mit Fragen verbunden ist, die auf die 
versöhnliche Justiz angewandt werden, scheint jedoch für viele noch neu zu sein (Guimarães, 2013).

Obwohl die Vermittlung im brasilianischen Rechtssystem seit der Zeit des Imperiums präsent ist, erlangte dieses Institut in den letzten Jahrzehnten Bekanntheit. Seit der politischen Emanzipation Brasiliens im Jahre 1822 hat die Verfassung des Imperiums offen die Lösung von Konflikten durch außergerichtliche Mittel gefördert, und die Schlichtung ist eine präventive und unabdingbare Voraussetzung für die Verarbeitung jeder Sache, wobei die Figur der Gerechtigkeit des Friedens das wichtigste Mittel zur Beilegung des Rechtsstreits ist (Silva, 2013).

1982 wurde das Gesetz der Sondergerichte für kleine Zwecke mit dem Ziel veröffentlicht, die Justiz auf nationaler Ebene zu entbürokratisieren. Gegründet 1984 dieses Gericht endgültig, durch Gesetz 7.244/84 (Sérpias, 2015).

Mit der Verabschiedung dieses Gesetzes hat die Schlichtung wieder eine Große Rolle bei der Konfliktlösung, einschließlich der Einfügung der Figur des Schlichters, die später untersucht wird (Guimarães, 2013).

Die Schlichtung ist eine Methode, die in weniger komplexen Konflikten angewandt wird, bei der der dritte Vermittler eine aktivere, aber neutralere Position in Bezug auf den Konflikt einnehmen kann, ohne die Unparteilichkeit zu verlieren. Es ist ein kurzer einvernehmlicher Prozess, der eine wirksame Lösung von Konflikten innerhalb der möglichen Grenzen der sozialen Beziehungen zwischen den beteiligten Parteien anstrebt (Sérpias, 2015).

Zusammenfassend ist "die Schlichtung ein Institut des Prozesses, weil es einer seiner Momente ist, der Verfahrenseffekte erzeugt, das Wissbildprinzip und die Rolle des dazwischenliegenden Richters bei der Bildung des Willens der Parteien privilegiert" (Nassif, 2005).

Die Schlichtung ist ein selbstgefälliger Prozess, bei dem ein unparteiischer Dritter (der Schlichter) die Konfliktparteien bei der Suche nach einer zufriedenstellenden Lösung für alle Parteien unterstützt (Silva, 2013). 
Die Schlichtung vor dem 4. Zivilgericht ist sehr weit entwickelt und praktiziert, da ein solches Gericht national für die Entwicklung und Anzahl der positiven Abstimmungen bekannt ist. Die meisten der bereits eingereichten Fälle sind das Ergebnis der Genehmigung von Vereinbarungen, sowohl gerichtlich als auch außergerichtlich (Serra, 2015).

Wir können dann zu dem Schluss kommen, dass das Vermittlungsinstitut zweifellos das am weitesten verbreitete und bekannteste alternative Mittel zur Konfliktlösung in Brasilien ist, das es der Justiz ermöglicht, ihre Forderungen schnell zu lösen und die Möglichkeit einer friedlichen Lösung von Liden Wirklichkeit werden zu lassen.

\section{VERMITTLUNGSSCHRITTE}

Die Vermittlung, so informell sie auch sein mag, hat einige Schritte, die der Schlichter beim Aufbau eines Abkommens beachten muss, Schritte, die keine Regeln sind. Die Einhaltung dieser Schritte trägt jedoch zur Geschwindigkeit und Effektivität des Prozesses bei (Sérpias, 2015).

Daher müssen bei der Vermittlung Leitlinien eingehalten werden, und sie sollte nicht durch den einfachen Willen der Parteien oder des Schlichters erfolgen. Im Folgenden werden wir die wichtigsten Phasen des Vermittlungsverfahrens erwähnen.

\section{DIE VERSÖHNLICHE UND IHRE EIGENSCHAFTEN IM VIRTUELLEN ZIVILGERICHT}

Die interessante Frage zu diesem Zeitpunkt ist die Figur des Schlichters in den Verfahren des Gesetzes 9.099/95. Schlichter werden vorzugsweise von Rechtsstudenten rekrutiert. Wenn das Gesetz also am liebsten gesagt hat, dann macht es es nicht zur Pflicht. So können Rechtswissenschaftler auch als Schlichter eine Figur machen. Gemäß Gesetz 9.099/95: "Art. 7. Die Schlichter und Laienrichter sind Hilfskräfte der Justiz, rekrutiert, der erste, vorzugsweise unter den Jurastudenten, und der zweite, unter Anwälten mit mehr als fünf Jahren Erfahrung" (Brasilien, 1995). 
In der Praxis sind die überwiegenden Mehrheit der Schlichter des Sonderzivilgerichts Jurastudenten, die in der Regel nach Eintritt ins fünfte Semester von Universitäten gewonnen werden. Als Partnerschaft, in der man Arbeit gegen Erfahrung tauscht, nicht anders im 4. Gericht des Zivilgerichts (Serra, 2015).

Der Schlichter ist eines der Themen der Vermittlung und muss neutral und unparteiisch handeln, um einen Moment und eine Situation zu schaffen, in deren Parteien sie sich wohl fühlen können, zu verhandeln. Er muss seine Psychologie nutzen, um die Vermittlung fruchtbar zu machen, durch den Willen der Parteien auf den entgegengesetzten Seiten, das heißt, der Schlichter ist nichts anderes als ein Vermittler (Porto, 2008).

Selbst wenn er die ganze emotionale Last trägt, muss der Schlichter während der Schlichtungsverhandlung so unparteiisch wie möglich und neutral sein und die Geschmeidigkeit und Legitimität des Prozesses gewährleisten (Silva, 2013). Die Unparteilichkeit des Schlichters ist für die Vermittlung von grundlegender Bedeutung, d. h. er wird keine der Parteien begünstigen, geschweige denn ein Urteil im Zusammenhang mit dem Konflikt. Sie sollte daher vertrauen an die Parteien weitergeben. Nur so ist es möglich, ein faires Abkommen für alle zu schließen (Amaral, 2011).

In Bezug auf das 4. Gericht des besonderen Zivilgerichts, sehr berühmt für die Anzahl der fruchtbaren Versöhnungen, in täglichen Anhörungen oder Veranstaltungen, die sporadisch wie: staatliche oder nationale Schlichtung Woche und "Samstag ist auch konzililiar tag", zeichnet sich für die Effizienz seiner Schlichter, und die meisten sind Auszubildende der höheren Ebene des Rechts, angesichts ihrer Partnerschaften zwischen dem Gericht selbst und dem TJAP unterzeichnet, Fecomércio, FEMICRO und SEBRAE (Serra, 2015).

Daher ist die Figur des Schlichters angesichts der Vermittlung von grundlegender Bedeutung, denn er ist derjenige, der die Arbeit organisiert und durchführt. Sie muss im Einklang mit dem zu beachtenden Recht und den Richtlinien handeln. 


\section{DIE EFFIZIENZ DER SCHLICHTUNG}

Nicht nur die mangelnde Effektivität und Schnelligkeit sind die Hauptprobleme der Justiz, andere Situationen tragen dazu bei, die Justiz zu verzögern und zu stoppen. Daher haben sich durch die Bundesverfassung von 19888, die durch das Gesetz 9.099/95 geregelt ist, spezielle Zivilgerichte als eine Möglichkeit erwiesen, den Zugang zur Justiz zu erweitern und wurde als "Fluchtventil" zur gemeinsamen Justiz charakterisiert, indem traditionelle Verfahren geändert wurden, die insbesondere zur Lösung kleiner Konflikte unwirksam waren (Guimarães, 2013).

Die Schaffung von Sondergerichten und der Einsatz von Schlichtung hat eine Wirksamkeit, die weit über das erwartete Maß hinausgeht, denn sie hat nicht nur die Justiz entlastet, sondern auch bürgerlich in den Geltungsbereich gerückt, die bis dahin diese Mittel nicht zur Lösung ihrer Konflikte genutzt haben, wodurch das Recht und die Kultur des Friedens verbreitet wurden (Guimarães, 2013).

So hat sich die Vermittlung im Laufe der Jahre als wirksam erwiesen, da sie die Justiz durch die Senkung von Kosten, Fristen und die Lösung von Ursachen weniger kompliziert entfache und neue Parameter für die Konfliktlösung in der Justiz insgesamt unter Berücksichtigung der Geschwindigkeit geschaffen hat (Guimarães, 2013).

So basiert die höhere Geschwindigkeit der Sonderrichter auf der Idee von Schnelligkeit und Effektivität, und es gibt zwei Möglichkeiten, Effizienz zu unterteilen, qualitativ und quantitativ. Das Quantitative betrifft nicht die Verfahrensqualität, wobei nur die Geschwindigkeit der Verfahren und die Kostensenkung zu berücksichtigen sind, während qualitativ im Gegenteil der Inhalt der Entscheidungen unter Berücksichtigung ihrer Qualität in Bezug auf die Einhaltung und Annahme des demokratischen Prozesses mit allen ihm innewohnenden Normen berücksichtigt wird (Neto, 2015).

In diesem Sinne können wir sagen, dass, obwohl Sondergerichte und Schlichtung wirksam sind, die Beschleunigung ihrer Verfahren nicht bedeutet, dass die Probleme der Justiz gelöst sind, geschweige denn sagen können, dass die Gerechtigkeit als Ganzes schnell ist (Barouche, 2010). 
In Bezug auf Macapa sind besondere Richter sehr effektiv, da der Anreiz zur Schlichtung gegeben ist (Serra, 2015).

Zum Beispiel hat der 4. Gerichtshof des Sondergerichtshofs von Macapa, ehemaliger Hof für Kleinstunternehmen und Kleinunternehmen, eine sehr konkrete und effektive Arbeit durch Vermittlung, national für seine Arbeit anerkannt, die jährlich zu fruchtbaren Versöhnungen führt (Serra, 2015).

Im Laufe des Jahres werden zahlreiche Vermittlungsaktionen durchgeführt, wie zum Beispiel staatliche und nationale Vermittlungswochen. In innen ist der 4. Gerichtshof des Sonderzivilgerichts von Macapá immer als das, das die fruchtbarsten Versöhnungen macht (Serra, 2015).

Der physische Teil des Gerichts begrenzt zweifellos die Zahl der Schlichtungen erheblich, aber trotzdem finden täglich 12 bis 25 Schlichtungsverhandlungen statt, eine Zahl, die im Vergleich zu den anderen Sondergerichten des Zentralgerichts von Macapá eine höhere Zahl ist (Serra, 2015).

Abbildung 1- zeigt die Anzahl der Fälle in Bezug auf Schlichtung, Weisung und Urteil

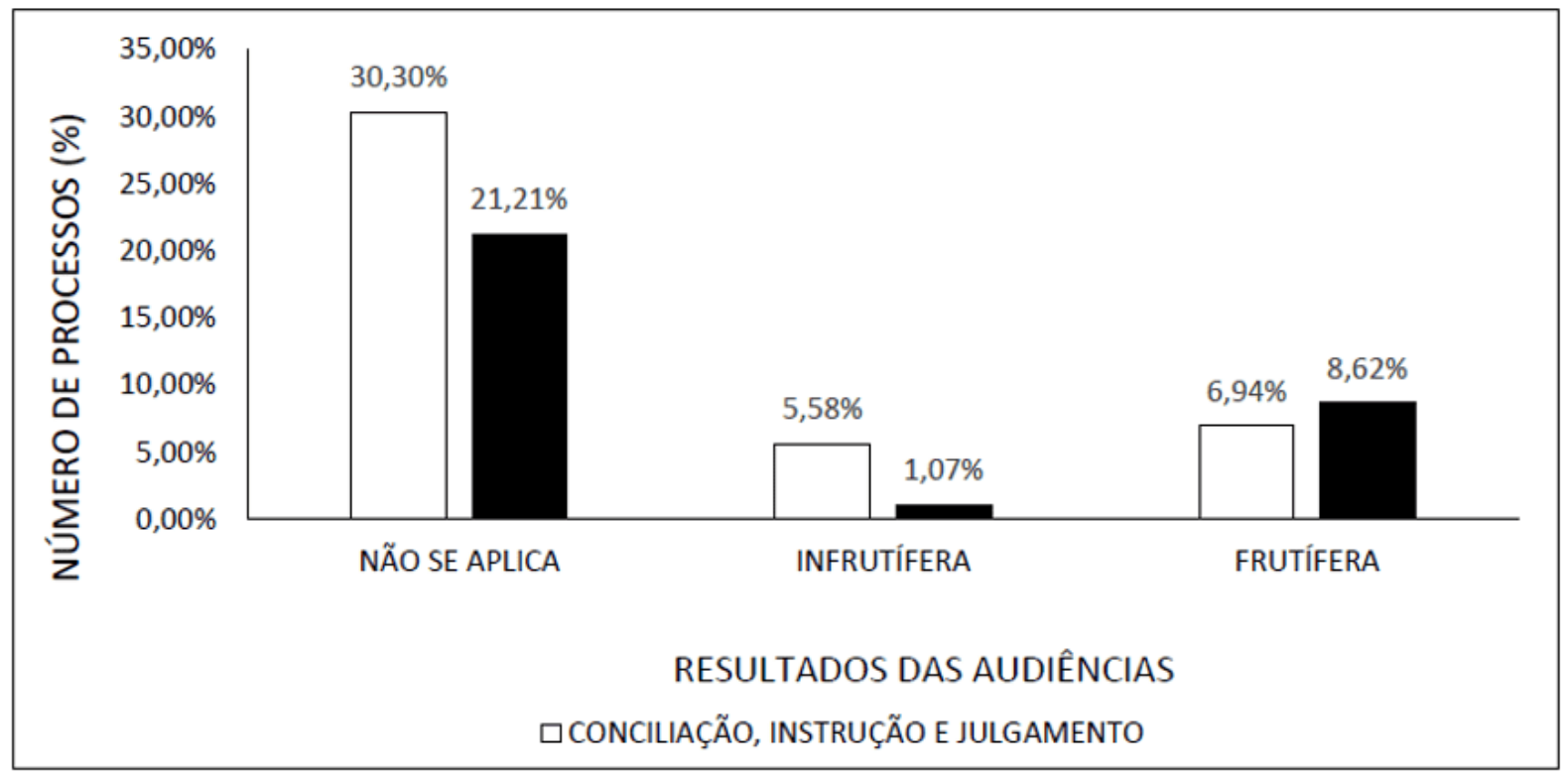

(Serra, 2015). 
Abbildung 1 zeigt die Effizienz der Schlichtungsverhandlungen, die vor dem 4. Gericht des Zivilgerichts von Macapá abgehalten werden. Während der ersten Vermittlungsanhörungen ist der Prozentsatz der fruchtbaren Vereinbarungen höher; und die erfolglose Vereinbarung ist kleiner (angesichts der Anwesenheit des Schlichters), in Bezug auf das Stadium der Anweisung und des Urteils, vor dem Richter.

Daher können wir zu dem Schluss kommen, dass das Ziel der Schaffung von Sondergerichten vor dem 4. Gericht des Zivilgerichts von Macapá erreicht wurde, da durch die Vermittlung die Anzahl der durch Vereinbarungen gelösten Forderungen im Verhältnis zu den anderen Verfahrensphasen erheblich ist. Damit ist die Wirksamkeit des Schlichters als Motor der Schlichtungsverhandlungen zu zeigen.

\section{SCHLUSSFOLGERUNGEN}

Angesichts der steigenden Zahl von Fällen scheint die Justiz überlastet und ineffizient geworden zu sein, und es ist notwendig, nach neuen Mitteln zur Konfliktlösung zu suchen. Die Antwort der Justiz war die Schaffung von Sondergerichten durch Vermittlung.

Es hat eine historische Entwicklung der Sonderjustizgegebenen gegeben, von ihrer Umsetzung über das Gesetz 9.099/95 bis zur Konstruktion ihrer Prinzipien wie Verfahrensschnelligkeit, Informalität und, alles deutet auf die Realisierung eines schnelleren und effizienteren Prozesses hin.

PROJUD und TUCUJURIS durch die Computerisierung von Spezialgerichten scheinen wichtig zu sein, um den Prozess zu modernisieren, sie einfacher, schneller und zugänglicher für alle zu machen und damit globalen technologischen Trends zu folgen.

Das Schlichtungsinstitut erweist sich als wichtigstes alternatives Mittel zur Konfliktlösung, als eine Form der Selbstzusammensetzung, mit der die Parteien die Lösung der Probleme schaffen und die Justiz schnell ertränken. 
Die Vermittlung durch Schnelligkeit, niedrige Kosten und Effizienz scheint die Justiz für einen Teil der gesellschaft mit niedrigem Einkommen und für die Laien in diesem Bereich attraktiver zu machen, die an die Möglichkeit glauben, ihre Probleme hinter der Gerechtigkeit zu lösen und so positive Auswirkungen auf die soziale Befriedung zu erzeugen.

Die Entwicklung des Sonderverfahrens auf der Grundlage des Schlichtungsverfahrens wäre ohne die Anwesenheit des Schlichters nicht wirksam. Er ist es, der die Parteien an der Ausarbeitung eines Abkommens anweist, unparteiisch führt und das Verfahren nach zahlreichen Grundsätzen und Leitlinien leitet. Daher sollte die Ausbildung dieser Agenten stärker in den Vordergrund gerückt werden, da sie bei den meisten Forderungen der Justiz an vorderster Front stehen, die direkt und täglich mit der Bevölkerung und ihren Problemen in Stresssituationen und manchmal schwer zu vereinbaren sind.

Sondergerichte als Ganzes sollten versuchen, immer mehr in Schlichter und Schlichtungsklagen $\mathrm{zu}$ investieren, wie etwa staatliche und nationale Vermittlungswochen, denn so wird es eine große Möglichkeit geben, dass Fälle in der Justiz stärker fließen.

\section{VERWEISE}

AMARAL, R. L. S. D. A Concilição no Processo Judicial, . 2011. 64 (Graduação). Faculdade de Ciências Jurídicas da Universidade Tuiuti do Paraná, Curitiba PR.

BACELLAR, R. P. Juizados Especiais - a Nova Mediação Paraprocessual. São Paulo: Revista dos Tribunais, 2003. 286.

BAROUCHE, T. D. O. Os Juizados Especiais Cíveis e a Problemática da Celeridade Processual. Revista de Direito dos Monitores da UFF, n. 7, p. 1-24, 2010.

BRASIL. LEI № 9.099, DE 26 DE SETEMBRO DE 1995. JURÍDICOS, S. P. A. Brasília DF: Presidência da República - Casa Civil 1995. 
CARVALHO, J. P. C. D. A Era Virtual do Processo Judicial: A Experiência dos Juizados Especiais Virtuais e o Projeto de Lei de Informatização do Processo. Revista Direito e Liberdade, v. 3, n. 2, p. 453 - 484, 2006.

GUIMARÃES, A. A Crise do Judiciário e a Eficácia da Conciliação nos Juizados Especiais Cíveis Estaduias de São Luis - MA. Revista Thesis Juris, v. 2, n. 1, p. 142 161, 2013.

NASSIF, E. Conciliação judicial e indisponibilidade de direitos: paradoxos da “justiça menor" no processo civil e trabalhista. São Paulo SP: Editora LTR, 2005.

NETO, A. G. A Audiência de Conciliação no Novo Código de Processo Civil. Revista Eletrônica de Direito Processual - REDP. 16, n. 16, p. 29-57, 2015.

NETO, F. D. C. T.; JÚNIOR, J. D. F. Juizados Especiais Estaduais Cíveis e Criminais. Comentários à Lei no 9.099/95. 7ª̣ed. São Paulo: Revista dos Tribunais, 2011. 960p.

PORTO, A. P. G. Juizados Especiais Cíveis - O Papel do Conciliador 2008. 59p. (Especialização). Universidade Estadual Vale do Acaraú, Fortaleza CE.

SALOMÃO, L. F. As Microempresas nos Juizados Especiais. Revista da EMERJ, v. 4, n. 4, p. 62-73, 2001.

SENA, A. G.; RICHA, M. D. A. A conciliação judicial trabalhista em uma política pública de tratamento adequado e efetivo de conflito de interesses. In: PELUSO, A. C. e RICHA, M. D. A. (Ed.). Conciliação e mediação: estruturação da política judiciária nacional. Rio de janeiro RJ: Forense, 2011. p.115-139.

SÉRPIAS, L. Juizado Especial, Cível, Criminal, Federal e da Fazenda Pública. São Paulo: TradeBook, 2015. 1615p.

SERRA, A. B. C. S. Juizado Especial da Microempresa e Empresa de Pequeno Porte: Um Estudo de Caso na Cidade de Macapá. 2015. 18p. (Graduação). Estácio de Sá, Macapá AP. 
SILVA, É. B. Conciliação Judicial. Brasília DF: Gazeta Juridica, 2013. 432p.

SILVA, P. G. A Conciliação como Instrumento de Solução de Conflitos. 2012. (Graduação). Centro Universitário Eurípides de Marília - UNIVEM, Marília SP.

TJAP. Sistema TUCUJURIS - Processo Judicial Eletrônico. Macapá AP, 2016. Disponível em: < http://www.tjap.jus.br/portal/sobre.html >. Acesso em: 22/02/2016.

TOLENTINO, A. V. A competência do Juizado Especial Cível. 2011. Disponível em: $<\quad$ http://www.direitonet.com.br/artigos/exibir/6387/A-competencia-do-JuizadoEspecial-Civel >. Acesso em: 30/05/2016.

Eingereicht: Mai 2021.

Genehmigt: Mai 2021. 